\title{
Full Endoscopic Posterior Cervical Foraminotomy in Management of Foraminal Disc Herniation and Foraminal Stenosis
}

\author{
Asrafi Rizki Gatam ${ }^{1-3}$ \\ Luthfi Gatam $^{\text {I-3 }}$ \\ Phedy ${ }^{1,2}$ \\ Harmantya Mahadhipta ${ }^{2,4}$ \\ Omar Luthfi ${ }^{2,3}$ \\ Ajiantoro ${ }^{2}$ \\ Syafrudin Husin ${ }^{2}$ \\ Dina Aprilya (iD ${ }^{5}$ \\ 'Orthopedic Spine Division, Fatmawati \\ General Hospital, Jakarta, Indonesia; \\ ${ }^{2}$ Orthopaedic and Spine Center Gatam \\ Institute, Eka Hospital BSD, Banten, \\ Indonesia; ${ }^{3}$ Orthopaedic spine surgeon, \\ Premier Bintaro Hospital, Banten, Indonesia; \\ ${ }^{4}$ Orthopaedic spine surgeon, Tangerang \\ General Hospital, Banten, Indonesia; \\ ${ }^{5}$ Orthopedic and Traumatology, Faculty of \\ Medicine Universitas Indonesia, Jakarta, \\ Indonesia
}

\begin{abstract}
Background: The development of full endoscopic procedures enables surgeons to visualize the operative field very clearly. Posterior foraminotomy using endoscopy was developed as a minimally invasive procedure to reduce the complication of the anterior cervical approach and to preserve the segmental motion without decreasing the effectiveness of nerve decompression. Our aim is to evaluate the result of full endoscopic posterior cervical foraminotomy in our center. Methods: This is a prospective single-arm study of 65 foraminal disc herniation and foraminal stenosis patients that underwent full endoscopic posterior foraminotomy procedures. All patients were routinely observed for 12-months duration to evaluate Visual Analog Score (VAS) of the neck, arm, and modified Macnab criteria.
\end{abstract}

Results: Arm pain VAS decreased significantly compared with the pre-operation state $(\mathrm{p}<$ $0.001,0.034,0.001$ on immediate post-operative, 6-months follow-up, and 12-months follow-up, respectively) even though $6.15 \%$ of patients had hypesthesia on follow-up. There was no neck pain observed during 1 year follow-up, and modified Macnab criteria showed a good outcome following full endoscopic posterior foraminotomy.

Conclusion: Full endoscopic posterior foraminotomy provides good nerve decompression with all the benefits of endoscopic spine procedure.

Keywords: cervical spine, foraminal stenosis, herniated disc, cervical foraminotomy, endoscopic, minimally invasive

\section{Introduction}

Cervical radiculopathy caused by foraminal pathology either from disc herniation or degenerative narrowing of the foramen is a very common condition. ${ }^{1}$ Surgical management by anterior discectomy and fusion (ACDF) is still considered the gold standard of care. ${ }^{2,3}$ Although satisfactory outcomes can be achieved by ACDF, the risk of pseudoarthrosis, adjacent segment disease, loss of disc height, and approach-related complications such as dysphonia, dysphagia are sometimes cannot be avoided. ${ }^{4,5}$

Posterior procedures in the form of microscopic, endoscopic-assisted, or full endoscopic posterior foraminotomy are the other alternatives to ACDF for foraminal pathology. ${ }^{6}$ These procedures were developed to preserve mobility and stability of the cervical segment. However, the steep learning curves and the availability of the instrument were drawbacks that must be overcome by the attending surgeon. ${ }^{7}$

The aim of this paper is to evaluate the foraminal decompression procedure by full endoscopic technique, review the problem that the surgeon might encounter during the procedure, and evaluate the clinical result after the operation.
Correspondence: Dina Aprilya Eka Hospital BSD, Banten, Indonesia Email dina.cia.aprilya@gmail.com 


\section{Materials and Methods}

This is a prospective single-arm study from January 2018December 2019, all patients were followed up until 1-year post-operation. The inclusion criteria were 1 or 2 level degenerative foraminal stenosis or foraminal disc herniation without instability symptoms and with single-sided radicular arm symptoms. The exclusion criteria were myelopathic patients, loss of disc height, and concurrent cervical deformity. All patients had already undergone conservative treatment for at least 3 months with no clinical improvements. The conservative therapy consists of physical therapy and diagnostic root injection to confirm relief of symptoms and location of nerve impingement. During the 1-year follow-up, we found 65 patients eligible for full endoscopic posterior foraminotomy.

The evaluation of clinical results used the visual analog scale (VAS) of axial neck pain and arm pain, along with the modified Macnab criteria pre-operation and post-operation day one, 3 months, 6 months, and 12 months. Sensory disturbances in the form of hypesthesia and numbness, operation time, and hospital stay were also evaluated.

\section{Operative Technique}

The surgery was performed under general anesthesia in a prone position with a slightly reverse Trendelenburg position. We used the head holder instead of Mayfield clamps and placed the head in a slight flexion position. The arms were placed towards the caudal on the side with tension taping over the shoulder. Basically, the surgery follows the conventional posterior cervical foraminotomy procedure. $^{8,9}$

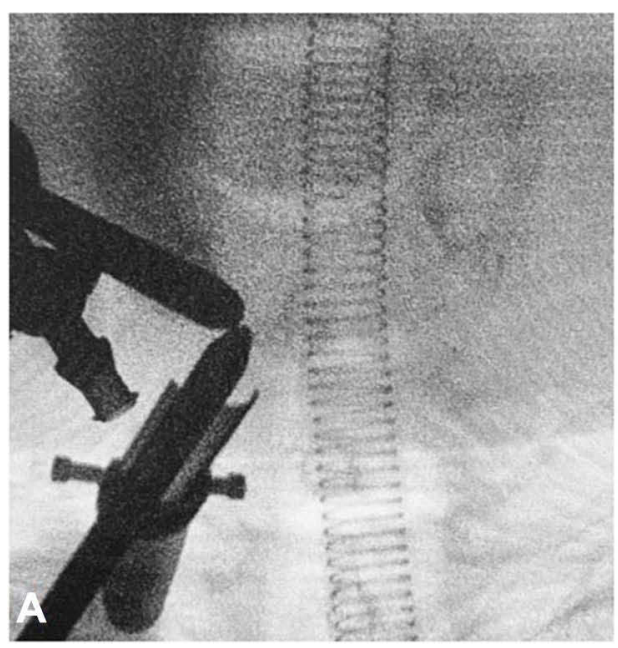

Marking of the incision site was done under fluoroscopic images (Figure 1). An anteroposterior view was used to mark the lamino-facet junction over the affected cervical foramen. Insertion of the obturator was done under lateral fluoroscopy to prevent too deep insertion of the obturator into the spinal canal which could damage the dura mater or even the myelum. The obturator was inserted until touching the lamino-facet junction, then the periosteum was stripped off the bone. The working channel was then inserted over the obturator.

Endoscopic procedure was started by identifying the V-point which was the medial border of the facet joint (Figure 2). Drilling was started from the inferior edge of superior lamina until medial part of inferior articular process (IAP) and superior articular process (SAP) (Figure 3). Superior edge of inferior lamina was also drilled until we can see the medial border of the pedicle. After all the bony work were finished, the flavum ligament were removed, at this point we should see the lateral border of the dura mater and venous plexus over the nerve root. Bipolar radiofrequency was used to coagulate venous plexus and prepare the nerve root (Figure 4). Discectomy was not routinely done; it was only performed if there was compression from the ventral side (See Supplementary Video 1 and Supplementary Video 2).

All data were calculated using IBM SPSS Statistic 25 with paired $T$ test and the relevant non-parametric statistics. A $p<0.05$ was considered statistically significant.

\section{Result}

A total of 65 eligible patients consists of 34 male patients and 31 female patients. The diagnosis was paracentral and foraminal disc herniation in 20 patients and degenerative foraminal

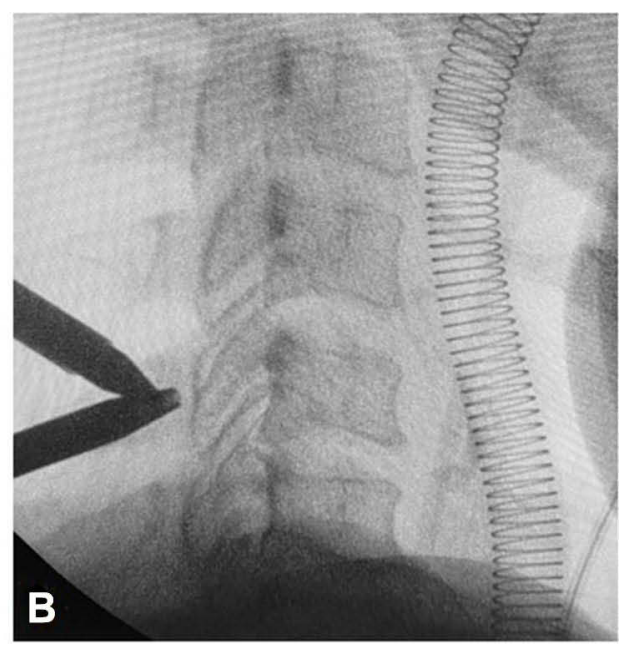

Figure I Marking the lamino-facet junction on affected level by image intensifier. (A) Anteroposterior view. (B) Inserting the obturator guided with lateral C-Arm image. 

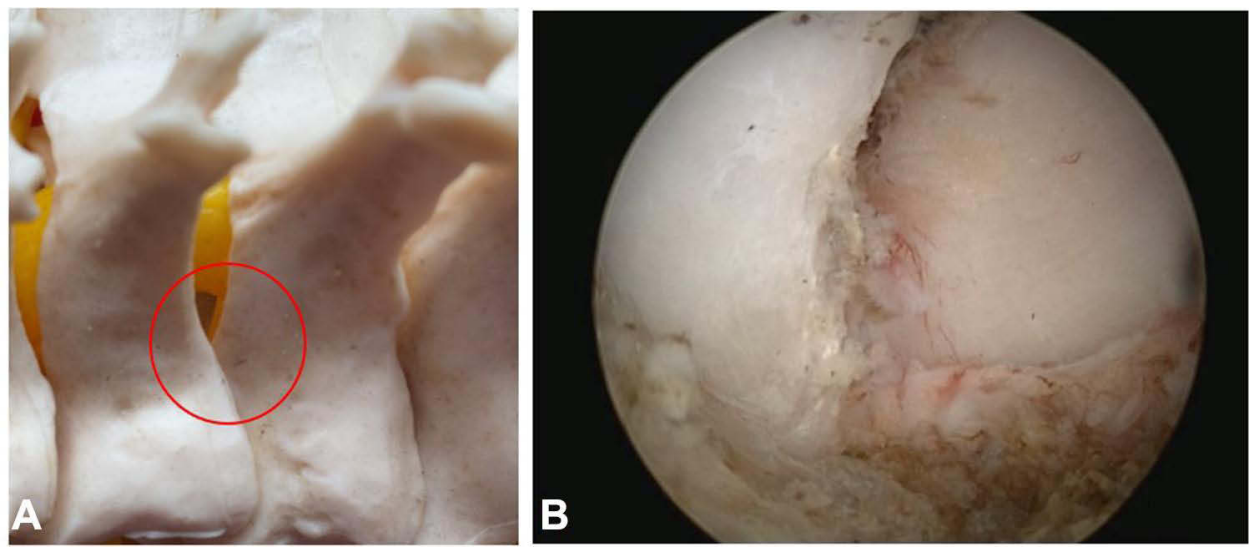

Figure 2 The V-point identification. (A) V-shaped medial border of facet joint (red circle). (B) The endoscopic view of V-point.
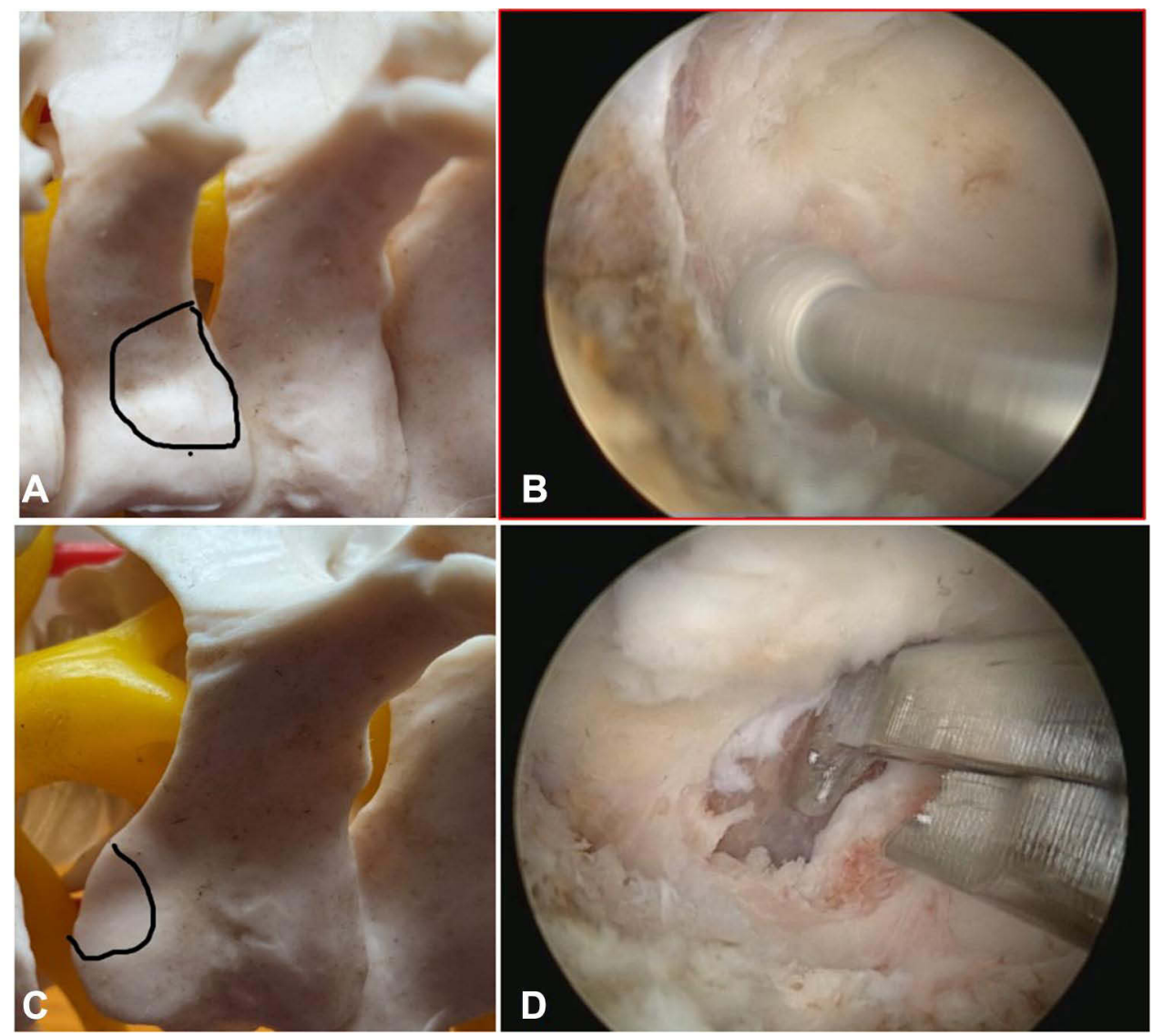

Figure 3 (A and B) Resection of the medial border of inferior articular process (IAP). (C and D) Superior articular process (SAP) medial resection and partial superior pediculectomy. Noted the black lines which encircled the medial border of the articular process.

stenosis in 45 patients. The mean age of the patients was 45.6 years old, with the age ranging from 33 years old to 78 years old. Younger patients in our study usually have disc herniation problems and older patients usually have bony stenosis problems. The affected level of the foraminal problem was as follows: 15 patients in C4-5 level, 29 patients in C5-6 level, and 21 patients in C6-7 level. The mean duration of surgery was 47.8 minutes. There were 5 patients with prolonged surgical time until around 100 minutes due to profuse bleeding. Hospitalization post-operation usually around 1.5 days, most of the patients were discharged on day one after the operation. We routinely insert drain after the procedure to evaluate the post-operation hematoma, the mean production after 24 hours was $23.6 \mathrm{~mL}$ (Table 1). 

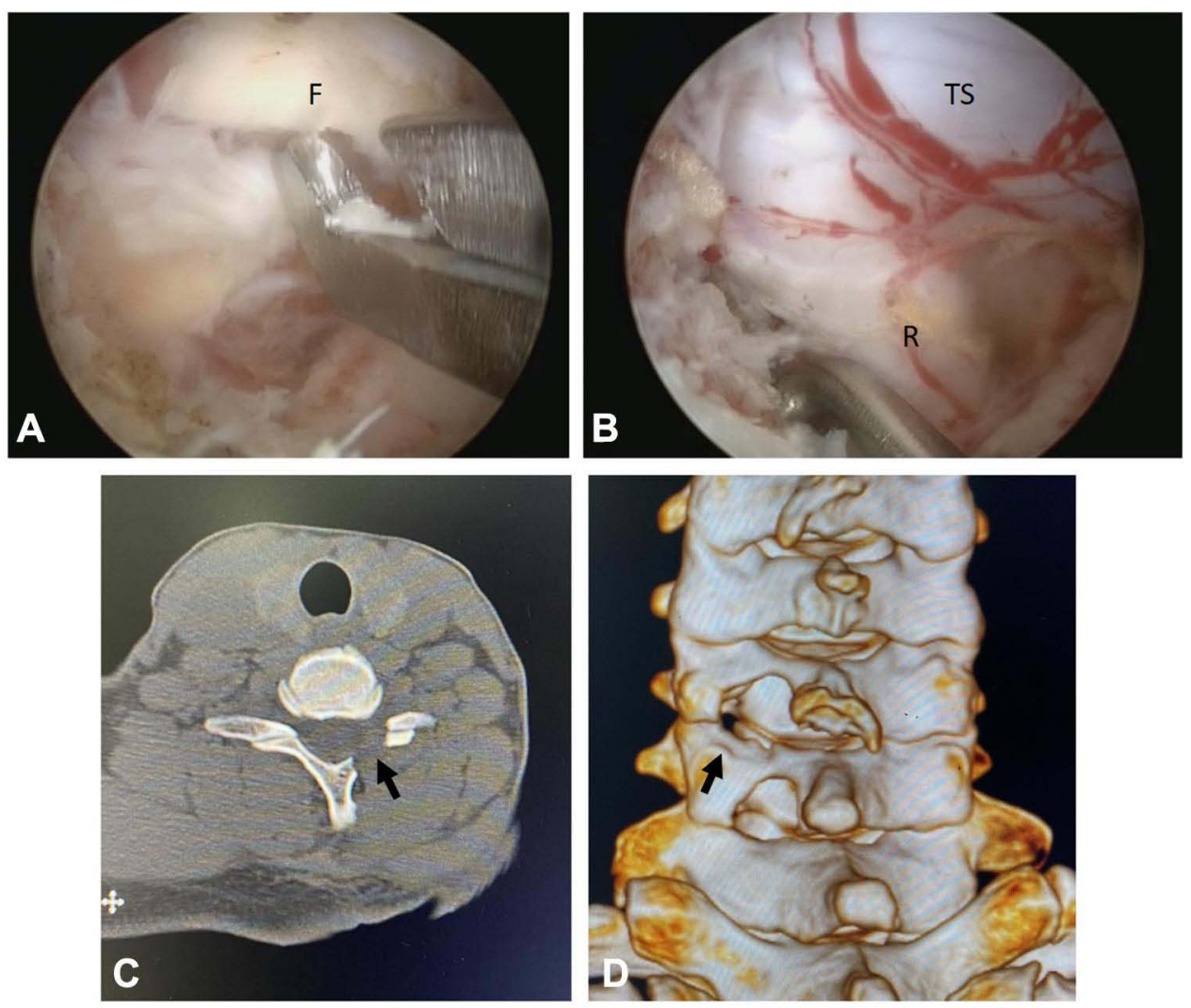

Figure 4 (A) Flavectomy. (B) Root mobilization and discectomy. (C) Post foraminotomy 2D axial CT Image. (D) Post foraminotomy 3D CT Image. Black arrows point on decompression site.

Abbreviations: F, Flavum; TS, Thecal Sac; R, Root.

The visual analog scale of the arm decreased significantly from a mean of 5 to a mean of 2 after the procedure, there were 4 patients with hypesthesia after the procedure (Figure 5A). Hypesthesia occurs in patients with disc herniation probably due to root retraction in order to

Table I Patient Characteristics

\begin{tabular}{|l|l|}
\hline Characteristic $\mathbf{( N = 6 5 )}$ & Value \\
\hline Age & $45.6(33-78)$ \\
\hline M:F & $34: 31$ \\
\hline Operation Level & 15 \\
\hline C45 & 29 \\
\hline C56 & 21 \\
\hline C67 & $47.8(30-100)$ \\
\hline Surgery Duration (mins) & $23.6(10-48)$ \\
\hline 24 h-post surgical drain production (mL) & $1.5(1-2)$ \\
\hline Length of Stay (day)
\end{tabular}

Note: Values are presented as number, mean (range), or number. remove disc material. All patients did not have significant axial neck pain on follow-up (Figure 5B).

Modified Macnab criteria showed 26 patients with excellent results without subsequent radicular pain, 34 patients good results with some occasional radicular symptoms but did not disturb daily living or work. Five patients resulted in fair and poor outcomes, these patients had hypesthesia and burning pain on the arm after the procedure. The symptoms were improved during followed up but did not resolve completely.

\section{Discussion}

Cervical radiculopathy caused by foraminal pathology either from disc herniation or degenerative narrowing of the foramen is a very common condition. ${ }^{1}$ Surgical management by anterior discectomy and fusion (ACDF) is very successful and still considered as the gold standard of care. ${ }^{2,3}$ Although satisfactory outcomes can be achieved by ACDF, the risk of pseudoarthrosis, adjacent segment disease, loss of disc height, and approach-related complications such as dysphonia, dysphagia are sometimes cannot be avoided. ${ }^{4,5}$ 

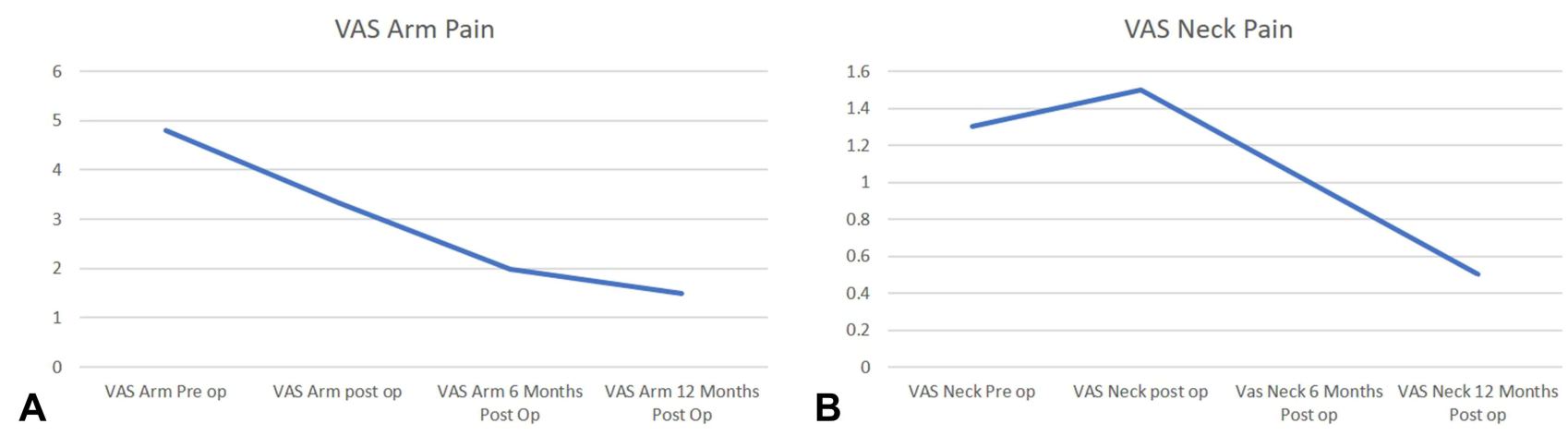

Figure $\mathbf{5}$ The Visual Analog Score (VAS) reduction. (A) VAS arm pain. There was a significant decrease on serial post-operative follow up until the last follow up. (B) VAS axial neck pain. There was slight increase in immediate post-operative period due to the post-operative wound and then the score reduced until the latest follow up.

Posterior procedures in the form of microscopic, endoscopic-assisted, or full endoscopic posterior foraminotomy are the other alternatives to ACDF for foraminal pathology. ${ }^{6}$ These procedures were developed to preserve mobility and stability of the cervical segment. ${ }^{7}$ The indications for this procedure are bony foraminal stenosis due to degenerative disease and foraminal and paracentral disc herniation. Posterior approach to the foramen is not without complication, axial neck pain, profuse bleeding, root injury, and dural injury are some of the problems surgeons might encounter during and after the procedure. ${ }^{7}$

Although conventional foraminotomy procedure provides a good result for decompression of the foramen, development of minimally invasive techniques in the form of full endoscopic technique has been developed to encounter risk of complications. ${ }^{8,9}$ Segmental stability can be preserved by reducing excessive bone resection in the facet joint and by reducing the paravertebral muscle injury around the neck. Preservation of stability will reduce postoperation axial neck pain and reduce the possibility of adjacent segment disease in the future. Furthermore, epidural fibrosis which can cause troublesome neurologic symptoms can also be prevented by the endoscopic spine procedure. $^{10,11}$

Developments of optics, surgical instruments such as endo-Kerrison punch, scissors, forceps, and various types of endoscopic burr allow the surgeon to decompress the foramen using the full endoscopic method very effectively. ${ }^{1,9}$ Endoscopic view provides an image with a magnification greater than the microscope. Thus, it could reduce complications to the nerve structure due to the closer location of the lens to the surgical field. Furthermore, venous bleeding can be properly reduced due to the continuous flow of water irrigation with appropriate $^{\text {pressure. }}{ }^{8}$
Posterior foraminotomy is a procedure to widen the size of the foramen through a minimally invasive approach. ${ }^{10}$ This technique has several advantages over anterior procedure such as direct visualization of the nerve structure and the fact that fusion is not necessary, this avoids the possibility of adjacent segment disease and loss of motion segment. ${ }^{11}$ This is shown by research from Ruetten et al, which on followed up there was no instability after the procedure. ${ }^{1}$

There are differences in several techniques for posterior foraminotomy using microscopic tubular retractor, endoscopic assisted tubular retractor, and full endoscopic method. ${ }^{9,12}$ The first is the medium, the first two procedure uses air as the medium, but the full endoscopic technique uses water as the medium. Water pressures can directly control bleeding over the operative field, whereas by using air the surgeon has to continuously control the bleeding using coagulation. ${ }^{11}$ Secondly, the high mobility of the full endoscopic technique enables the surgeon to evaluate and see clearly the nerve structure for adequate decompression, whereas the other two uses lever arm to fix the tube into the operating table which creates a relatively fixed tube location over the facet joints. ${ }^{13}$ The learning curves of all these procedures are different, the full endoscopic technique has a steeper learning curve compared with the tubular technique, the possibility of nerve injury is higher in the early learning curve of full endoscopic technique.

Full endoscopic spine surgery is not without disadvantages. One of the main problems is the very steep learning curve, the surgeon has to be very familiar with the endoscopic view which is a two-dimensional image and they need to have a lot of experience in managing simple lumbar discectomy or simple lumbar decompression before jumping into full endoscopic cervical foraminotomy procedure. ${ }^{12}$ The other problem is the availability of the instrument which cannot be easily accessed by every surgeon. 
The effectivity of posterior foraminotomy had already been evaluated by several studies. Ahn et al found good to excellent results in $92 \%$ of cases, whereas MacDowall et al found in $93 \%$ of patients. Several studies also showed good resolution of radiculopathy symptoms after 5 years followed up. ${ }^{3,4}$ This is consistent with the result of this study that most of the patients had resolution of symptoms with good to excellent modified Macnab criteria and decreasing of arm VAS after the procedure.

Several studies evaluated the efficacy of posterior foraminotomy to anterior cervical discectomy and fusion (ACDF), most of the research showed the result between the two procedures were equal. ${ }^{14,15}$ The revision rate did also not differ between both procedures. Several surgeons did not really agree to compare posterior foraminotomy and ACDF because they think that ACDF is not a minimally invasive procedure. We think posterior foraminotomy is comparable with non-fusion anterior foraminotomy procedure, in which several studies showed that anterior foraminotomy resulted in the loss of lordosis and disc height but had a lower blood loss compared to the posterior procedure. ${ }^{5}$

The move toward minimal invasiveness and endoscopy on the other cervical pathology has also been reported in some other studies. Yang et $\mathrm{al}^{16}$ proved the safety and efficacy of micro-endoscopic laminoplasty in cervical myelopathy patients. Fully Endoscopic decompression for cervical myelopathy has also been studied by Yadav et $\mathrm{al}^{17}$ and proved to be a safe and effective alternative treatment option in selected patients. These findings show that the full endoscopic decompression is not only expanding the range of options for patients with radiculopathy but also for patients with degenerative cervical myelopathy. ${ }^{18}$

\section{Conclusion}

Full endoscopic posterior foraminotomy with or without discectomy is highly effective for the management of cervical radiculopathy. This full endoscopic procedure has a low reoperation rate and reduces the need for post-operation analysis. However, a randomized control study is needed to further evaluate the outcome of this procedure.

\section{Ethics Approval and Consent to Participate}

This research had already been approved by ethical committee in Fatmawati General Hospital Jakarta and Eka Hospital, Tangerang. The privacy and personal identity information of all participants were protected in accordance with the Declaration of Helsinki. Written informed consent has been obtained from all participants.

\section{Consent for Publication}

My manuscript does not contain any individual person data. The co-authors consented to the publication of this article.

\section{Acknowledgements}

Dina Aprilya is now affiliated with Eka Hospital BSD, Banten, Indonesia.

\section{Funding}

This research did not receive any specific grant from funding agencies in the public, commercial, or not-for-profit sectors.

\section{Disclosure}

The authors declare that they have no competing interest.

\section{References}

1. Ruetten S, Komp M, Merk H, Godolias G. Full-endoscopic cervical posterior foraminotomy for the operation of lateral disc herniations using 5.9-mm endoscopes: a prospective, randomized, controlled study. Spine (Phila Pa 1976). 2008;33(9):940-948. doi:10.1097/ BRS.0b013e31816c8b67

2. Foster MT, Carleton-Bland NP, Lee MK, Jackson R, Clark SR, Wilby MJ. Comparison of clinical outcomes in anterior cervical discectomy versus foraminotomy for brachialgia. $\mathrm{Br} J$ Neurosurg. 2019;33(1):3-7. doi:10.1080/02688697.2018.1527013

3. Ahn Y, Keum HJ, Shin SH. Percutaneous endoscopic cervical discectomy versus anterior cervical discectomy and fusion: a comparative cohort study with a five-year follow-up. J Clin Med. 2020;9(2):371. doi:10.3390/jcm9020371

4. MacDowall A, Heary RF, Holy M, Lindhagen L, Olerud C. Posterior foraminotomy versus anterior decompression and fusion in patients with cervical degenerative disc disease with radiculopathy: up to 5 years of outcome from the national Swedish Spine Register. J Neurosurg Spine. 2020;32(3):344-352. doi:10.3171/2019.9.SPINE19787

5. Cheung JPY, Luk KDK. Complications of anterior and posterior cervical spine surgery. Asian Spine J. 2016;10(2):385-400. doi:10.4184/asj.2016.10.2.385

6. Sahai N, Changoor S, Dunn CJ, et al. Minimally Invasive posterior cervical foraminotomy as an alternative to anterior cervical discectomy and fusion for unilateral cervical radiculopathy: a systematic review and meta-analysis. Spine (Phila Pa 1976). 2019;44 (24):1731-1739. doi:10.1097/BRS.0000000000003156

7. Skovrlj B, Gologorsky Y, Haque R, Fessler RG, Qureshi SA. Complications, outcomes, and need for fusion after minimally invasive posterior cervical foraminotomy and microdiscectomy. Spine $J$. 2014;14(10):2405-2411. doi:10.1016/j.spinee.2014.01.048

8. Bhatia S, Brooks NP. Posterior endoscopic cervical foraminotomy. Neurosurg Clin N Am. 2020;31(1):9-16. doi:10.1016/j.nec.2019.08.001

9. Ross DA, Bridges KJ. Technique of minimally invasive cervical foraminotomy. Oper Neurosurg. 2017;13(6):693-701. doi:10.1093/ons/ opx053

10. Park JH, Jun SG, Jung JT, Lee SJ. Posterior percutaneous endoscopic cervical foraminotomy and diskectomy with unilateral biportal endoscopy. Orthopedics. 2017;40(5):e779-e783. doi:10.3928/ 01477447-20170531-02 
11. Wagner R, Telfeian AE, Iprenburg M, Krzok G. Minimally invasive fully endoscopic two-level posterior cervical foraminotomy: technical note. J Spine Surg. 2017;3(2):238-242. doi:10.21037/ jss.2017.05.06

12. Ruetten S, Komp U, Merk H, Godolias G. A new full-endoscopic technique for cervical posterior foraminotomy in the treatment of lateral disc herniations using 6.9-mm endoscopes: prospective 2-year results of 87 patients. Minim Invasive Neurosurg. 2007;50 (4):219-226. doi:10.1055/s-2007-985860

13. Xi Z, Lu Y, Xie L. Endoscopic posterior cervical foraminotomy via a single stab incision for contiguous two-level cervical radiculopathy Acta Neurochir (Wien). 2020;162(3):685-689. doi:10.1007/s00701-01904198-4

14. Liu WJ, Hu L, Chou PH, Wang JW, Kan WS. Comparison of anterior cervical discectomy and fusion versus posterior cervical foraminotomy in the treatment of cervical radiculopathy: a systematic review. Orthop Surg. 2016;8(4):425-431. doi:10.1111/os.12285
15. Tong Y, Huang Z, Hu C, et al. A comparison study of posterior cervical percutaneous endoscopic ventral bony decompression and simple dorsal decompression treatment in cervical spondylotic radiculopathy caused by cervical foraminal and/or lateral spinal stenosis: a clinical retrospective study. BMC Musculoskelet Disord. 2020;21 (1):1-10. doi:10.1186/s12891-020-03313-2

16. Yang Y, Liu ZY, Zhang LM, et al. Microendoscopy-assisted minimally invasive versus open transforaminal lumbar interbody fusion for lumbar degenerative diseases: 5-year outcomes. World Neurosurg. 2018;116:e602-e610. doi:10.1016/j.wneu.2018.05.049

17. Yadav YR, Parihar V, Ratre S, Kher Y, Bhatele PR. Endoscopic decompression of cervical spondylotic myelopathy using posterior approach. Neurol India. 2014;62(6):640-645. doi:10.4103/0028-3886.149388

18. Ganau M, Holly LT, Mizuno J, Fehlings MG. Future directions and new technologies for the management of degenerative cervical myelopathy. Neurosurg Clin N Am. 2018;29(1):185-193. doi:10.1016/j.nec.2017.09.006
Orthopedic Research and Reviews

\section{Publish your work in this journal}

Orthopedic Research and Reviews is an international, peer-reviewed, open access journal that focusing on the patho-physiology of the musculoskeletal system, trauma, surgery and other corrective interventions to restore mobility and function. Advances in new technologies, materials, techniques and pharmacological agents are particularly
Dovepress

welcome. The manuscript management system is completely online and includes a very quick and fair peer-review system, which is all easy to use. Visit http://www.dovepress.com/testimonials.php to read real quotes from published authors. 